\title{
Polarization measurements of unusual cases of medium frequency burst emissions extending below $1.5 \mathrm{MHz}$
}

\author{
J. LaBelle* (1)
}

\begin{abstract}
Auroral medium frequency burst (MFB) is a radio emission of natural auroral origin associated with substorm expansion phase and observable at ground level. The emission usually occurs at frequencies above $1500 \mathrm{kHz}$, but occasionally it extends to a sharp lower cutoff frequency at 1300-1500 kHz depending on the observing site, with a frequency gap below the cutoff and sporadic emission below the gap extending to frequencies as low as $1000 \mathrm{kHz}$. These low-frequency MFB components lie below the electron gyrofrequency and hence could represent either whistler or LO-modes. Recently, using crossed antennas and a two-channel receiver at Toolik Lake, Alaska, polarization of these low-frequency MFB components was measured for the first time and found to be left-hand. This observation eliminates whistler mode as a possibility and requires the low-frequency components be LO-mode in the ionosphere, which constrains their source location since it requires that the frequency exceeds the L-cutoff frequency. In these occasional events marked by a cutoff and low-frequency MFB components, the latter probably originate at high altitudes $(>800 \mathrm{~km}$ ) and reach the ground through extraordinary low-density polar cap ionosphere.
\end{abstract}

Keywords: Auroral radio emission, Substorm, Substorm onset, Langmuir wave, Mode conversion radiation, Medium frequency burst

\section{Introduction}

Radio emissions detectable at ground level provide a window into auroral plasma physics processes and conditions. At least four types occur, and while all of them occur during substorms, two of them, medium frequency burst (MFB) and impulsive auroral hiss, stand out because of their specific association with the expansion phase (Makita 1979; LaBelle et al. 1994; reviews by Sazhin et al. 1993; LaBelle and Treumann 2002). MFB is a broadband left-hand polarized emission of typically a few minutes duration at substorm onset (Weatherwax et al. 1994; LaBelle et al. 1997, 2005; Sato et al. 2008). It is best observed from a station poleward of the onset location. Radio interferometry at such stations shows that the emission direction of arrival moves in parallel with the poleward-most expanding substorm arc (Bunch

\footnotetext{
*Correspondence: jlabelle@einstein.dartmouth.edu

Department of Physics and Astronomy, Dartmouth College, Hanover, NH, USA
}

et al. 2009), and observation of the emission ceases when the arc comes overhead of the station due to absorption of MFB signals in the enhanced D-region underneath the arc created by impact ionization from the auroral electrons.

Recent studies show MFB is not a featureless impulsive emission, as suggested by low-resolution measurements, but rather consists of complex fine structures, most commonly shifting downward in frequency by $100-300 \mathrm{kHz}$ over tens of ms (Bunch and LaBelle 2009). LaBelle (2011) put forth an explanation involving Langmuir wave excitation over a range of altitudes, hence range of plasma frequencies, in the topside auroral ionosphere. The group delay of the Langmuir waves, which depends on the magnitude of the downward spatial gradient in electron density, can explain time delays of $10 \mathrm{~s}$ of ms. A simple one-dimensional model suggests that relatively low-energy parallel electron beams are required for this mechanism to explain the observed delays, exactly the 
characteristics of Alfvenically accelerated electron beams which have been inferred to compose the polewardexpanding substorm arc based on IMAGE satellite data (Mende et al. 2003). If this is true, it implies that MFB and its complex structure provide a tool to remotely sense and study the temporal and spatial dynamics of the Alfvenic aurora. Subsequent investigations provide some support for the topside generation of MFB (e.g., Broughton et al. 2012), but the mechanism has not been proven and many uncertainties remain. One reason for these is absence of in situ data from the MFB source region due to its relatively small size, rapid motion, and few minutes duration which makes it nearly impossible to target with a rocket launch.

Lack of in situ data motivates a search for alternative remote sensing methods of probing the physics underlying MFB. In this regard, important clues may come from nulls and cutoffs which occasionally occur in the MFB spectrum. One of these, investigated in this paper, is a low-frequency cutoff near $1.5 \mathrm{MHz}$, depending on the observing location, which is sometimes accompanied by a burst of lower-frequency MFB emission below a frequency gap below the cutoff. Because this cutoff is near the electron gyrofrequency in the ionosphere above the observing station, LaBelle and Treumann (2002) speculate that the mechanism of MFB perhaps radiates into two different modes depending on frequency: LO-mode above the gyrofrequency and whistler mode below. Polarization measurements are needed to test this suggestion, and they have hitherto been unavailable, partly due to the rarity of the phenomenon. However, recently a polarization experiment tuned to the appropriate frequency range has been operated from Toolik Lake, Alaska. "Data presentation" section presents results of this experiment, and "Discussion" section presents some broader data analysis which contributes to interpreting the results.

\section{Data presentation}

Over the years, Dartmouth College has operated LF/MF/ HF receivers at various high-latitude sites in both the northern and southern hemispheres. Until recently, the bulk of these measurements was conducted with stepped frequency receivers having frequency and time resolutions of about $10 \mathrm{kHz}$ and $1 \mathrm{~s}$, respectively. In some cases, multiple antennas were deployed to measure polarization (e.g., Shepherd et al. 1997) or direction of arrival (e.g., Hughes et al. 2001; Bunch et al. 2008, 2009). In the past decade, techniques have changed radically with the advent of high-speed analog to digital conversion, software-defined radio, and large-capacity disk drives. For example, the most recent experiment deployed at Toolik Lake, Alaska, directly samples signals from six antennas at rates of $10-20 \mathrm{MHz}$ continuously for many hours per night, resulting in polarization and direction of arrival information with resolution generally limited only by the bandwidth and stationarity of the phenomenon. These experiments collect several terabytes of information daily and rely on regular monitoring, with full resolution data archived only for selected events.

Figure 1 shows six example spectrograms recorded with instruments described above at five high-latitude sites: Churchill, Manitoba $(58.7684 \mathrm{~N}, 94.1650 \mathrm{~W}, 69.12$ inv. lat.); Baker Lake, Nunavut (64.3176N, 96.0220W, 74.10 invariant); South Pole, Antarctica (90S, 74.25 inv. lat.); Sondrestrom, Greenland (66.9873N, 50.9448W, 72.85 inv. lat.); and Toolik Lake, Alaska (68.6318N, $149.6060 \mathrm{~W}$, 68.72 inv. lat.). The most prominent feature in each panel is MFB emission, in these cases spanning from below $1500 \mathrm{kHz}$ to as high as $3000 \mathrm{kHz}$. These examples have been selected to show a rare and unexplained property of MFB: when it extends to the lowest part of its normal $1500-4500 \mathrm{kHz}$ frequency range, it exhibits a distinct lower cutoff in the range $1300-1500 \mathrm{kHz}$, depending on site. This cutoff frequency is close to the electron gyrofrequency in the ionosphere above the observing site, as discussed below. Above the cutoff frequency, MFB appears as broadband impulsive emission on the time resolution of these measurements, although it has been shown to have complex fine structure when viewed at higher time resolution (Bunch and LaBelle 2009).

Below the $1300-1500 \mathrm{kHz}$ cutoff frequency, there is a frequency gap in which no MFB occurs. The width of this gap ranges from a few $\mathrm{kHz}$ to $>100 \mathrm{kHz}$ in the examples shown. Below this gap, intermittent bursts of MFB occur extending to frequencies as low as $1000 \mathrm{kHz}$. In every example, the MFB observed below the cutoff, compared to that above the cutoff, occurs for a much smaller fraction of the total duration of the event and is generally less intense.

Below $1000 \mathrm{kHz}$, impulsive LF auroral hiss is often observed, a whistler mode emission which like MFB occurs at substorm onsets (e.g., Makita 1979; Morgan 1977; LaBelle et al. 1994). In some cases, intense bursts of auroral hiss are correlated with the bursts of MFB below the $1300-1500 \mathrm{kHz}$ cutoff. The association of auroral hiss with MFB, and with bursts of MFB below the electron gyrofrequency in particular, as well as the existence of the distinctive cutoff and gap, motivates speculation that the portion of MFB below the gyrofrequency might be whistler mode. According to this scenario, the MFB generation mechanism produces either LO-mode or W-mode depending on the emission frequency (LaBelle and Treumann 2002, p. 406). In the absence of polarization measurements, this interpretation remained speculative.

The multi-antenna measurements conducted at Toolik Lake imply that for the examples in the bottom two 


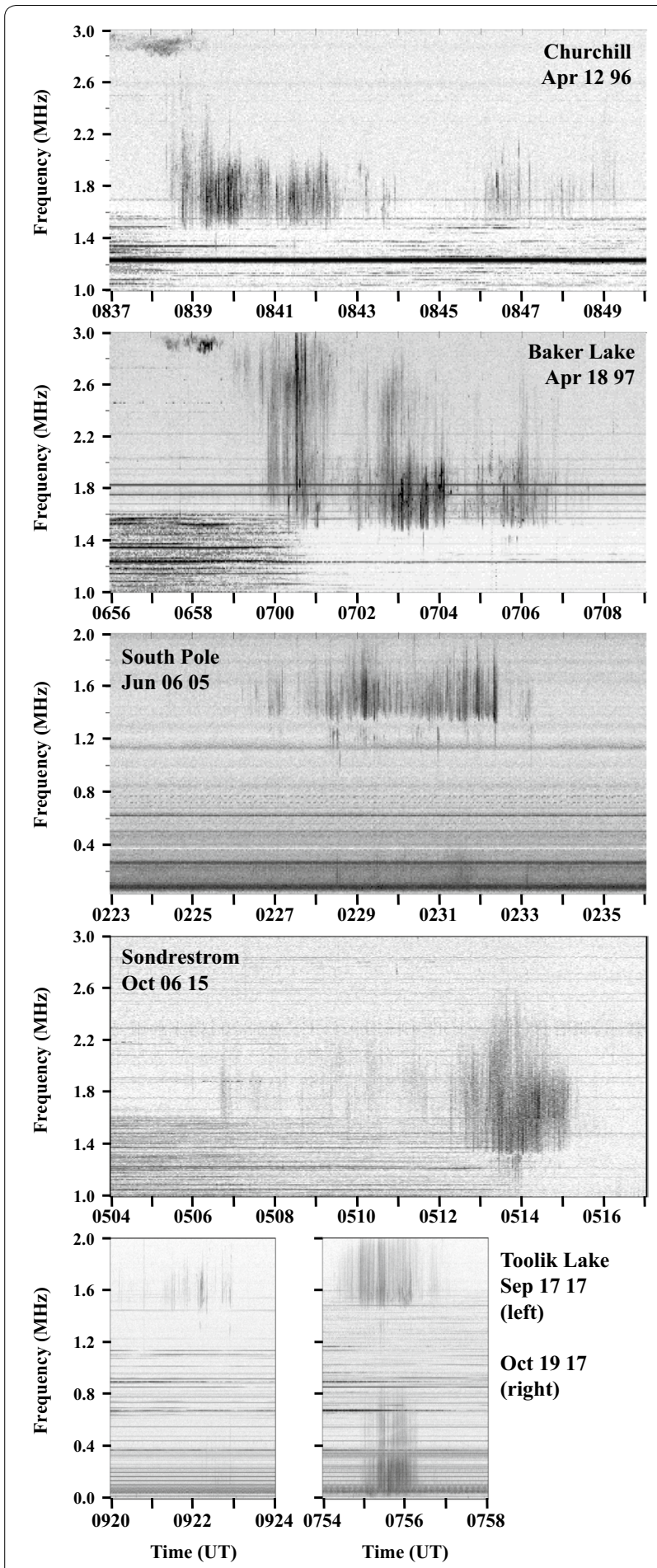

Fig. 1 Spectrograms showing six examples of MFB emissions observed at five sites. The events are all of an unusual type, in which the MFB has a distinct lower cutoff at 1.3-1.5 MHz, with a gap below, and sporadic emissions extending from below the gap to as low as 1.0 $\mathrm{MHz}$. In some cases, auroral hiss is observed below $1.0 \mathrm{MHz}$ panels of Fig. 1, polarization of the signals can be determined. This is done by measuring the phase difference, after correcting for variable cable lengths and delays in the electronics, between the MFB signals detected by two identical antennas oriented at $90^{\circ}$ to one another. Using cross-spectral analysis, this phase difference can be measured for each frequency component. Figure 2 shows the results for the event occurring at 0754-0758 UT on October 19, 2017. The bottom panel of Fig. 2 shows the power spectrum versus time, the same information as in the lower right panel of Fig. 1, but from a different antenna. The upper panel of Fig. 2 shows the phase difference which indicates the polarization, with blue and red representing ninety degree negative and positive phase differences, corresponding to right-hand and left-hand polarization, respectively. As expected, the MFB above the electron gyrofrequency is left-hand polarized, and the whistler mode hiss below the gyrofrequency is right-hand polarized. The surprising new result concerns the components of MFB below 1.5 MHz: these are clearly and unambiguously left-hand polarized, like the MFB above the cutoff and in contrast to the whistler mode hiss.

Figure 3 shows another example, 0920-0924 UT on September 17, 2017, in the same format as Fig. 2. This example confirms the result of Fig. 2 concerning the polarizations of the MFB signals above and below the frequency gap near the electron gyrofrequency: both are left-hand polarized, in contrast to the auroral hiss.

\section{Discussion}

The new result, demonstrating that the polarization of $<1.5 \mathrm{MHz}$ MFB emissions is left-hand, implies that these, like higher frequency MFB, propagate in the LOmode in the ionosphere, which constrains the source to locations where the MFB frequency exceeds the L-cutoff frequency $\left(f_{\mathrm{L}}\right)$. Figure $4 \mathrm{a}$ is a spectrogram of the event observed at Toolik Lake, 0754-0758 UT on October 19, 2017 (also shown in bottom right panel of Fig. 1 and bottom panel of Fig. 2). Figure $4 \mathrm{~b}$ shows, with the same frequency scale, altitude profiles of $f_{\mathrm{ce}}, f_{\mathrm{pe}}$, $f_{\mathrm{uh}}=\left(f_{\mathrm{pe}}^{2}+f_{\mathrm{ce}}^{2}\right)^{1 / 2}$, and $f_{L}=\left(\left(f_{\mathrm{ce}}^{2}+4 f_{\mathrm{pe}}^{2}\right)^{1 / 2}-f_{\mathrm{ce}}\right) / 2$. These are calculated using magnetic field from the International Geomagnetic Reference Field (IGRF), and electron density from the International Reference Ionosphere (IRI) Bilitza et al. (2011) for the Toolik Lake location and date of the observation.

The electron density from the IRI is not expected to be accurate for the ionosphere under the polewardexpanding substorm auroral arc which is the source the MFB. It may better represent density profiles in the quiet polar cap poleward of the arc, through which the MFB rays propagate to reach Toolik Lake. Interestingly, in this case the distinct lower cutoff of the MFB near $1.5 \mathrm{MHz}$ 


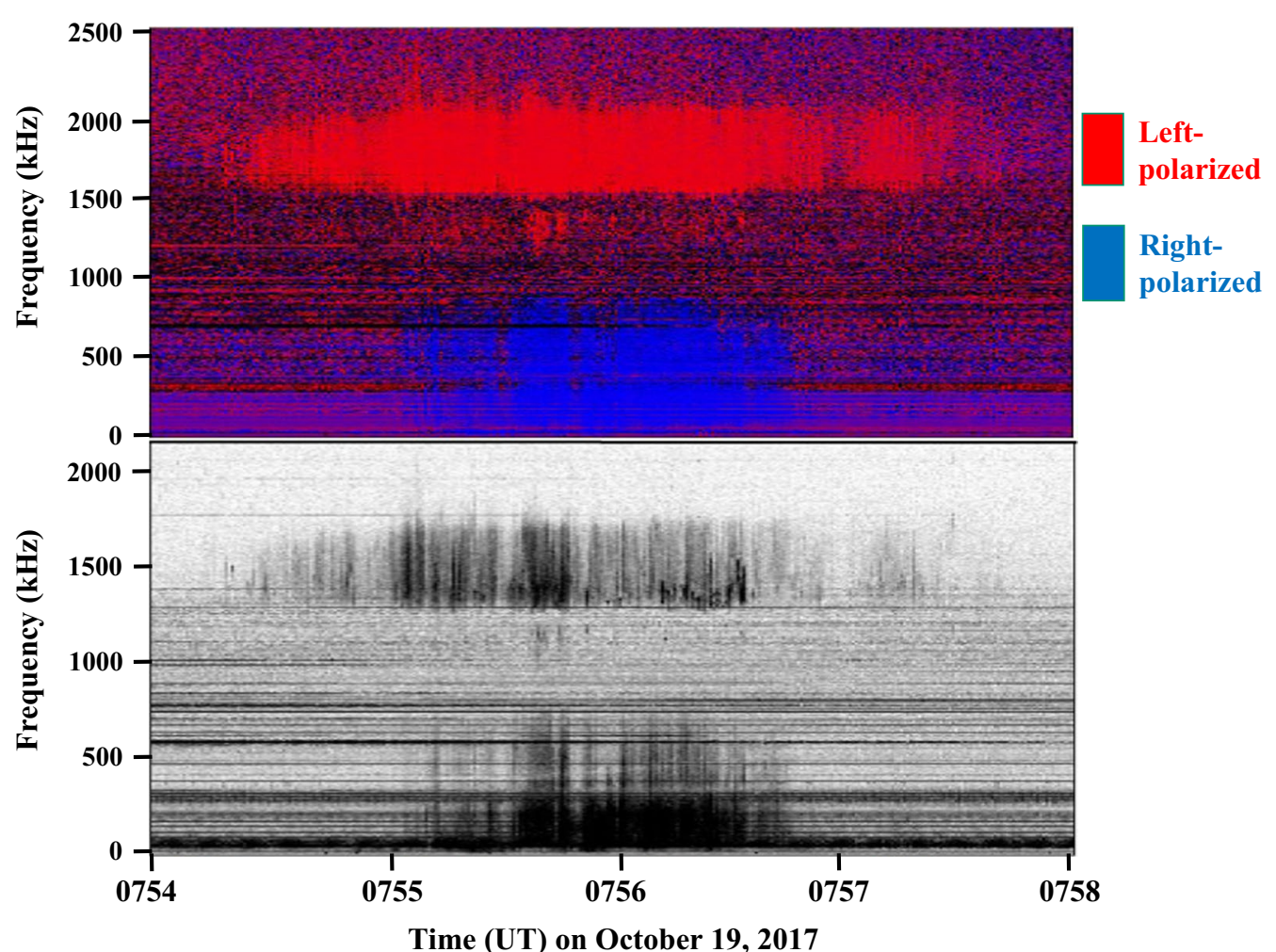

Fig. 2 Spectrograms of intensity (bottom panel) and polarization (top panel) of MFB emission observed at Toolik Lake on October 19, 2017, showing that the component of MFB below $1.5 \mathrm{MHz}$ is left-polarized, like the bulk of the MFB emission, and unlike the right-polarized whistler mode auroral hiss emission

corresponds approximately to the maximum value of the L-cutoff frequency in the ionosphere $\left(f_{\mathrm{L}, \max }\right)$, and the maximum observed frequency of MFB corresponds approximately to the maximum value of the plasma frequency in the ionosphere $\left(f_{\mathrm{pe}, \max }\right)$. If this is true, the bulk of the MFB at 1.5-2.0 MHz conforms to predictions of the topside generation mechanism proposed by LaBelle (2011), in which MFB originates as Langmuir waves generated over a range of altitudes at frequencies up to $f=f_{\text {pe,max }}$ and reaching the ground under the condition that $f>f_{\mathrm{L}, \max }$. Broughton et al. (2012) showed that a large number of MFB events observed at Sondrestrom, Greenland, obeyed this relationship.

Unfortunately, although this profile together with the generation mechanism proposed by LaBelle (2011) explains the bulk of the MFB at frequencies above $1.5 \mathrm{MHz}$ and may explain the majority of MFB events which contain only frequencies above about $1.5 \mathrm{MHz}$, it fails to account for the occasional occurrences of MFB below $1.5 \mathrm{MHz}$ in the Toolik Lake data shown in Fig. 4a. These $<1.5 \mathrm{MHz}$ components are now known to be L-polarized and hence must also propagate in the LO-mode above the L-cutoff in the ionosphere. An alternative scenario must therefore be developed for this particular MFB observation and the others like it.

The locations in Fig. $4 \mathrm{~b}$ where $f_{\mathrm{pe}}$ lies in the range 1.0$1.5 \mathrm{MHz}$ are at low altitudes $<250 \mathrm{~km}$ and high altitudes $>500 \mathrm{~km}$. The low-altitude source region can be eliminated because at these altitudes the emission frequencies would lie below the electron gyrofrequency. Below $f_{\text {ce }}$, the Landau resonant Langmuir waves couple naturally to the $\mathrm{W}$-mode not the $\mathrm{LO}$-mode, and the resulting radiation should be right-hand polarized. According to the electron density profile in Fig. 4b, the high altitude source region would suffer from the same problem, since the plasma frequency lies below the gyrofrequency in that region as well.

Furthermore, even if LO-mode signals below $1.5 \mathrm{MHz}$ could be produced in high altitude sources, according to Fig. $4 \mathrm{~b}$ they would lie below the maximum value of the L-cutoff frequency and hence could not penetrate to ground level. To explain penetration of LO-mode signals to ground level requires unusually low electron densities. Figure $4 \mathrm{c}$ shows a modified version of the IRI density profile, rescaled so that the maximum L-cutoff lies at 1.0 $\mathrm{MHz}$, low enough to allow the lowest left-polarized 


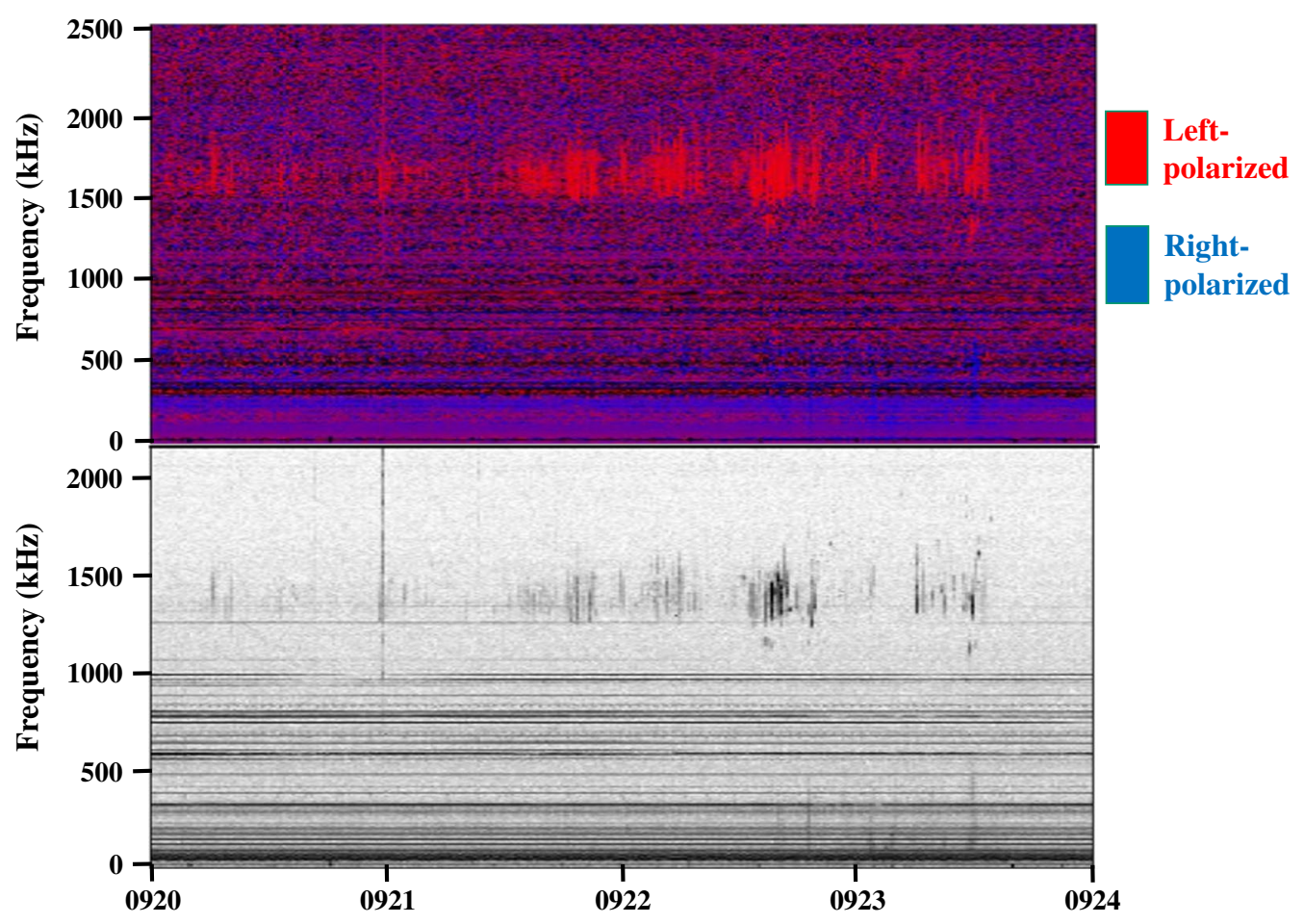

Time (UT) on September 17, 2017

Fig. 3 Spectrograms of intensity (bottom panel) and polarization (top panel) of MFB emission observed at Toolik Lake on September 17, 2017, confirming the result of Fig. 2 concerning polarization of the component of MFB below $1.5 \mathrm{MHz}$

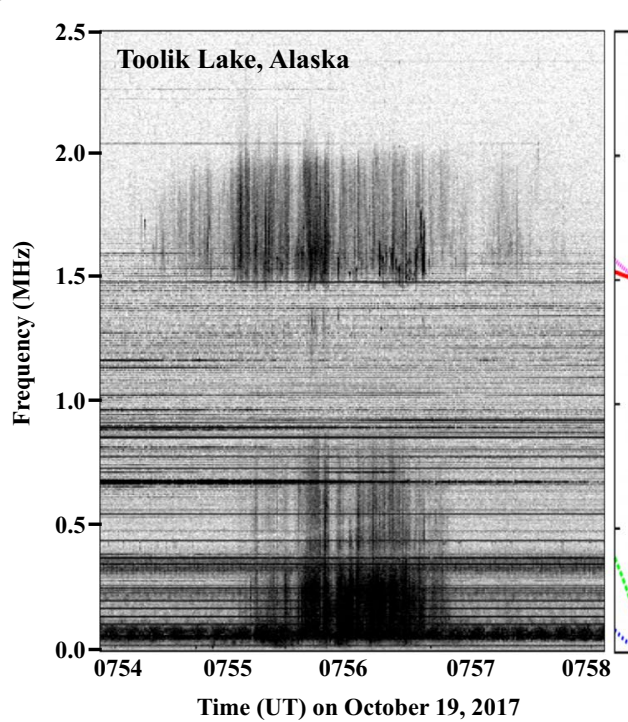

(a)

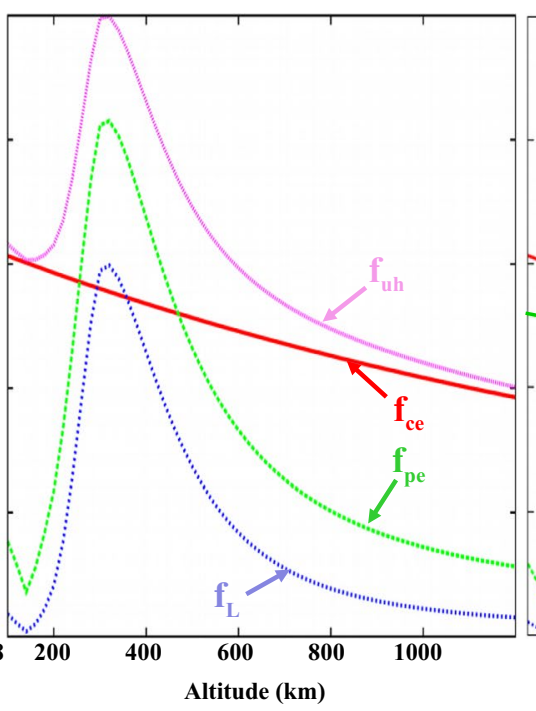

(b)

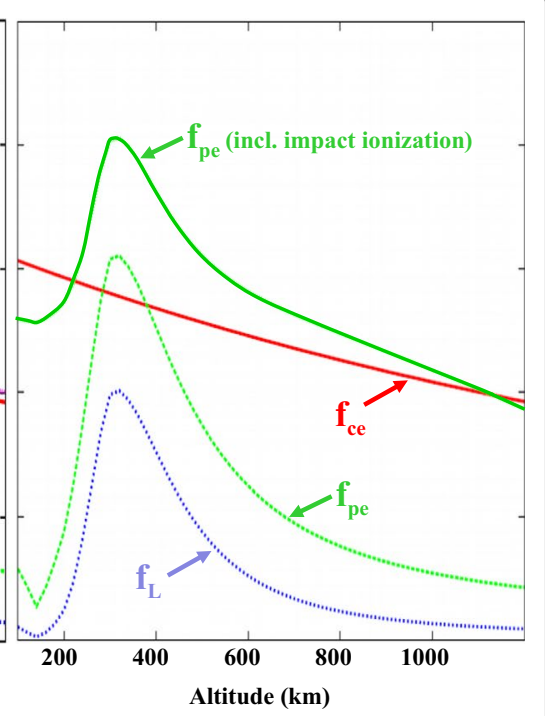

(c)

Fig. 4 a Spectrogram of intensity of MFB emissions observed at Toolik Lake on October 19, 2017; b altitude profiles of key frequencies $\left(f_{\text {ce, }} f_{\text {pe, }}\right.$ $f_{\mathrm{uh}}$, and $f_{\mathrm{L}}$ ) above Toolik Lake for the date and time of the observations in $\mathbf{a}$, based on IGRF and IRI model values of magnetic field and electron density; c altitude profiles of key frequencies calculated by scaling the IRI profile such that the maximum L-cutoff frequency lies below all observed left-polarized MFB, and plasma frequency profile (solid green trace) obtained by adding to the scaled-IRI model an enhancement approximating that expected from impact ionization due to low-energy auroral electrons 
signals observed at Toolik Lake to penetrate the ionosphere. In this case, the maximum plasma frequency is only $1.55 \mathrm{MHz}$, insufficient to explain the highest frequency MFB which extends to about $2 \mathrm{MHz}$. However, the electron density beneath the auroral arc should be enhanced due to electron impact ionization caused by the auroral electrons, including the same ones causing the MFB, which are suspected to be low-energy Alfvenically accelerated electrons. The solid green trace in Fig. 4c shows the plasma frequency profile assuming that the background low-density ionosphere (dashed green and blue profiles) is augmented by enhanced electron density peaked at $350 \mathrm{~km}$ and decreasing at higher altitudes with a roughly Gaussian roll-off of width $500 \mathrm{~km}$. This profile is meant to model the effects of electron impact ionization from low energy $(<100 \mathrm{eV})$ electrons: as seen in Fig. 14 of Strickland et al. (1983), for Gaussian electron spectra typical of discrete aurora, the electron density enhancement peaks somewhat above the maximum in the production rate, which lies near $300 \mathrm{~km}$ for $100 \mathrm{eV}$ electrons as shown by Fig. 3b of Fang et al. (2008). The Strickland model does not necessarily apply to the topside, where the recombination times are long, and transport processes can be significant in determining the density structure as discussed, for example, in Chapter 21 of Banks and Kockarts (1973). However, using the effect of impact ionization as a rough guideline, Fig. 4c shows an example profile with sufficient enhanced density to make the plasma frequency exceed the gyrofrequency up to about $1150 \mathrm{~km}$. The F-peak is also enhanced in this profile, which allows Langmuir waves as high as $2.0 \mathrm{MHz}$, as well as those low as $1.0 \mathrm{MHz}$, to convert to LO-mode electromagnetic waves and reach the ground through the lower-density ionosphere poleward of the arc. Unusually, low electron density in the polar cap combined with enhanced density on the field line of the auroral arc can therefore account for the observations of left-polarized MFB spanning 1-2 $\mathrm{MHz}$ at Toolik Lake. The need for extraordinary low density may explain the rarity of these low-frequency MFB which have been observed approximately monthly in Fall 2017 with the high-resolution instrument at Toolik, and generally less frequently over the years with lower-resolution instruments at other sites.

Toolik Lake direction of arrival (DOA) measurements may lend some support to this interpretation. For the event on Oct. 19, 2017, these data show that the lowfrequency MFB components come from slightly higher elevation angles than the bulk of the $>1500 \mathrm{kHz}$ MFB, suggesting that they may come from higher altitudes. DOA data from the Sep. 17, 2017, event show the lowfrequency and high-frequency MFB coming from approximately the same elevation angles, however. DOA at these frequencies is strongly affected by ionospheric refraction, so statistical analysis including ray-tracing consideration of many examples would be needed to confirm whether the $<1500 \mathrm{kHz}$ MFB components consistently come from higher altitudes than the normal $>1500 \mathrm{kHz}$ MFB components, as suggested by the Oct. 19, 2017, example.

This scenario leaves unexplained the narrow gap in the MFB just below 1.3-1.5 MHz depending on location. Electron density profiles measured with the Sondrestrom radar during MFB show the highest density in the E-region (e.g., Figs. 4c and 4d in Broughton et al. 2012) consistent with characteristics of other published electron density profiles associated with expanding substorm arcs (e.g., Fig. 1 of Oyama et al. 2014). One idea is that such profiles could have an inflection point in the topside density profile, though the enhanced electron density profile in Fig. 4c does not show this effect. Such an inflection point in the electron density profile would occur at approximately the same altitude where the Langmuir frequency would match the gap in the MFB emission. It is not clear how an inflection point would cause an interruption in MFB generation, however. Another possibility is that a trough in electron density above the density maximum could be deep enough to cause the plasma frequency to lie below the gyrofrequency for a short span of altitudes. Neither the modeled density profile of Fig. 4c nor the measured profiles in Broughton et al. (2012) or Oyama et al. (2014) show this effect, but if it occurred, it would explain the gap in the MFB spectrum since LOmode emission would not occur from the range of altitudes for which $f_{\text {ce }}>f_{\text {pe }}$ where Langmuir waves connect to the whistler mode as described above. In summary, there is no satisfactory explanation of the cutoff and gap in the MFB spectrum, but the new-found knowledge that the MFB below the gap, like that above it, is left-polarized places a significant constraint on whatever mechanism causes it.

The possible role of the electron gyrofrequency in explaining the frequency gap, and indeed the proximity of the MFB cutoff at $1300-1500 \mathrm{kHz}$ to the electron gyrofrequency, inspire the question of how the cutoff frequency varies with magnetic field. Figure $5 \mathrm{a}$ shows the average cutoff frequency at each of five sites, determined from Fig. 1, as a function of the geomagnetic field strength at an altitude of $100 \mathrm{~km}$ at each site, determined from IGRF. Figure $5 \mathrm{~b}$ shows the altitude range of the upper and lower boundaries of the gap in MFB below the cutoff frequency, assuming these match the electron gyrofrequency above the station, with the latter inferred from IGRF. Dashed lines in Fig. 5a indicate the gyrofrequency at altitudes of $100-400 \mathrm{~km}$, as a function of the magnetic field at $100 \mathrm{~km}$. The data points 

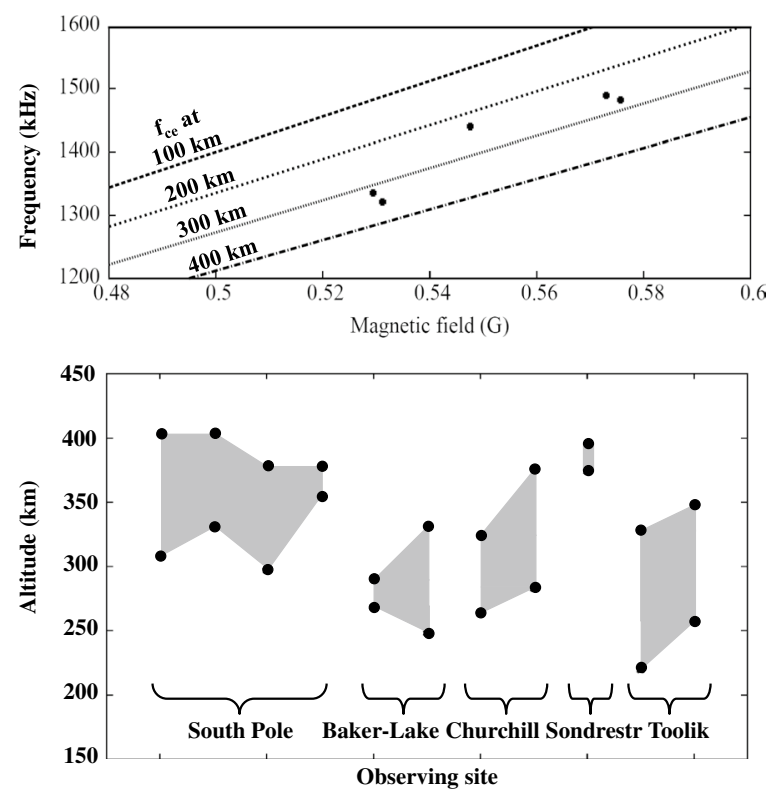

Fig. 5 a The average value of frequency of the lower cutoff of MFB emission, as a function of the magnetic field strength $100 \mathrm{~km}$ above the observing station. The frequency increases with magnetic field strength. Values of the electron gyrofrequency at altitudes of 100$400 \mathrm{~km}$ are indicated for reference. $\mathbf{b}$ Altitudes where the electron gyrofrequency matches the top and bottom frequency of the gap in MFB emissions, for selected spectra at each observing site

suggest a possible relationship between the cutoff frequency and the magnetic field, with larger magnetic field implying higher cutoff frequency. If associated with the gyrofrequency, the significant altitude range would be 250-350 km, which would be near the F-peak. If the cutoff corresponds to $f_{\mathrm{L}, \max }$, then electron density as well as magnetic field would play a role in determining the data points in Fig. 5a.

In summary, the main result of this paper is the determination that occasional MFB observed below a 1300$1500 \mathrm{kHz}$ frequency cutoff is left-hand polarized. This observation puts an end to speculation that such components, being below the ionospheric electron gyrofrequency, might be whistler rather than LO-mode. The requirement that they are LO-mode puts restrictions on their source altitude, where the emission frequency must exceed the L-cutoff frequency. The low-frequency components probably come from high altitudes, 800$1200 \mathrm{~km}$, where it is possible for both the emission frequency and the plasma frequency to exceed local $f_{\text {ce }}$ and maximum $f_{L}$. The MFB frequency gap below the 1300 $1500 \mathrm{kHz}$ cutoff remains unexplained. The cutoff frequency roughly scales with the magnetic field as expected if it is at least partially related to the gyrofrequency. The scenario described above to explain Toolik Lake observations could be further tested by statistical studies of direction of arrival measurements of MFB emissions exhibiting the cutoff, frequency gap, and low-frequency component.

\section{Authors' contributions}

$J \mathrm{~L}$ upgraded and calibrated the antenna array and receiving system in Toolik Lake during field trips in 2015 and 2017. JL monitored and archived Toolik Lake data as it was collected since 2015. JL analyzed the data used in this paper, interpreted the results, and wrote the paper. All authors read and approved the final manuscript.

\section{Acknowledgements \\ The author wishes to thank engineers Mike Trimpi, Hank Harjes, and David McGaw for their contributions to the receiving systems that collected the data shown in this paper. Thanks is also due to personnel at each observing site who contributed to operating the experiments, and to former Dartmouth graduate students Allan Weatherwax, Simon Shepherd, John Hughes, Nick Bunch, Matt Broughton, and Spencer Hatch, as well as Dartmouth under- graduates, who contributed to maintaining and operating these experiments over the years.}

\section{Competing interests}

The author declares that he has no competing interests in this manuscript.

\section{Availability of data and materials}

The data used in this study are archived at Dartmouth College and available on request by emailing jlabelle@einstein.dartmouth.edu.

\section{Funding}

This work was supported by the US National Science Foundation through Grants AGS-1147699 and PLR-1443338 to Dartmouth College.

\section{Publisher's Note}

Springer Nature remains neutral with regard to jurisdictional claims in published maps and institutional affiliations.

Received: 7 March 2018 Accepted: 18 August 2018

Published online: 04 September 2018

\section{References}

Banks PM, Kockarts G (1973) Aeronomy, part A. Academic Press, New York Bilitza D, McKinnell L-A, Reinisch B, Fuller-Rowell T (2011) The international reference ionosphere today and in the future. J Geod 85:909-920

Broughton M, LaBelle J, McCready M, Bunch N, Roberg-Clark G (2012) Experimental tests of a topside generation mechanism for auroral medium frequency radio emissions. J Geophys Res 117:A12309. https://doi. org/10.1029/2012JA018034

Bunch NL, LaBelle J (2009) Fully resolved observations of auroral medium frequency burst radio emissions. Geophys Res Lett 36:L15104. https://doi. org/10.1029/2009-GL-038513

Bunch NL, LaBelle J, Weatherwax AT, Hughes JM (2008) Auroral medium frequency burst radio emission associated with the March 23, 2007, THEMIS study substorm. J Geophys Res 113:A00C08. https://doi. org/10.1029/2008-JA-013503

Bunch NL, LaBelle J, Weatherwax AT, Hughes JM (2009) Experimental tests of the generation mechanism of auroral medium frequency burst radio emissions. J Geophys Res 114:A09302. https://doi.org/10.1029/2008JA-013993

Fang X, Randall CE, Lummerzheim D, Solomon SC, Mills MJ, Marsh DR, Jackman CH, Wang W, Lu G (2008) Electron impact ionization: a new parameterization for $100 \mathrm{eV}$ to $1 \mathrm{MeV}$ electrons. J Geophys Res 113:A09311. https://doi.org/10.1029/2008JA013384

Hughes JM, LaBelle J, Watermann J (2001) Statistical and case studies of $2 f_{c e}$ auroral roar observed with a medium-frequency interferometer. J Geophys Res 106:21147 
LaBelle J (2011) An explanation for the fine structure of MF burst emissions. Geophys Res Lett 38:L03105. https://doi.org/10.1029/2010-GL-046218

LaBelle J, Treumann RA (2002) Auroral radio emissions, 1. Hisses, roars, and bursts. Space Sci Rev 101:295-440

LaBelle J, Weatherwax AT, Trimpi ML, Brittain R, Hunsucker RD, Olson JV (1994) The spectrum of LF/MF/HF radio noise at ground level during geomagnetic substorms. Geophys Res Lett 21:2749

LaBelle J, Shepherd SG, Trimpi ML (1997) Observations of auroral medium frequency burst emissions. J Geophys Res 102:22221

LaBelle J, Weatherwax AT, Tantiwiwat M, Jackson E, Linder J (2005) Statistical study of medium-frequency burst emissions observed at South Pole Station and at multiple Canadian observatories. J Geophys Res 110(A2):A02305. https://doi.org/10.1029/2004-JA-010608

Makita K (1979) VLF/LF hiss emissions associated with aurora. Mem Nat Int Polar Res 16:1

Mende SB, Carlson CW, Frey HU, Peticolas LM, stgaard N (2003) FAST and IMAGE-FUV observations of a substorm onset. J Geophys Res 108(A9):1344. https://doi.org/10.1029/2002JA009787

Morgan MG (1977) Auroral hiss on the ground at $L=4$. J Geophys Res 82:2387
Oyama S, Miyoshi Y, Shiokawa K, Kurihara J, Tsuda TT, Watkins BJ (2014) Heightdependent ionospheric variations in the vicinity of nightside poleward expanding aurora after substorm onset. J Geophys Res Space Phys 119:4146-4156. https://doi.org/10.1002/2013JA019704

Sato Y, Ono T, lizima M, Kumamoto A, Sato N, Kadokura A, Miyaoka H (2008) Auroral radio emission and absorption of medium frequency radio waves observed in Iceland. Earth Planets Space 60:207-217. https://doi. org/10.1186/BF03352783

Sazhin SS, Bullough K, Hayakawa M (1993) Auroral hiss: a review. Planet Space Sci 41:153-166

Shepherd SG, LaBelle J, Trimpi ML (1997) The polarization of auroral roar emissions. Geophys Res Lett 24:3161

Strickland DJ, Jasperse JR, Whalen JA (1983) Dependence of auroral FUV emissions on the incident electron spectrum and neutral atmosphere. J Geophys Res 88(A10):8051-8062. https://doi.org/10.1029/JA088iA10p 08051

Weatherwax AT, LaBelle J, Trimpi ML (1994) A new type of auroral radio emission at 1.4-3.7 MHz observed from the ground. Geophys Res Lett 21:2753

\section{Submit your manuscript to a SpringerOpen ${ }^{\circ}$ journal and benefit from:}

- Convenient online submission

- Rigorous peer review

- Open access: articles freely available online

- High visibility within the field

- Retaining the copyright to your article

Submit your next manuscript at $\boldsymbol{\nabla}$ springeropen.com 